\title{
PARP15 expression associates with survival in triple negative breast cancer.
}

Shahan Mamoor, $\mathrm{MS}^{1}$

${ }^{1}$ shahanmamoor@gmail.com

East Islip, NY USA

We mined published microarray data (1) to understand the most significant gene expression differences in the tumors of triple negative breast cancer (TNBC) patients based on survival at time of analysis: dead or alive. The poly (ADP-ribose) polymerase family, member 15, PARP15, emerged as among the most differentially expressed genes, transcriptome-wide, when comparing the primary tumors of triple negative breast cancer patients dead or alive. PARP15 mRNA was present at significantly higher quantities in the tumors of TNBC patients alive. Importantly, PARP15 expression was significantly correlated with overall survival in basal subtype breast cancer, a molecular subtype sharing significant overlap with triple negative breast cancer. PARP15 may be of relevance as a biomarker or as a molecule of interest in understanding the etiology or progression of triple negative breast cancer.

Keywords: triple negative breast cancer, TNBC, systems biology of breast cancer, targeted therapeutics in breast cancer, PARP15, poly (ADP-ribose) polymerase family, member 15. 
Triple negative breast cancer is defined by lack of expression of the receptors for the hormones progesterone and estrogen (PR negative and ER negative), as well as for the human epidermal growth factor receptor 2 (HER2 negative) $(2,3)$. Though not completely identical, triple negative breast cancer shares significant overlap with the basal or basal-like molecular subtype of human breast cancer (3). TNBC is more frequently diagnosed in women of African descent (black women) for reasons not understood, and their disease course is similarly more aggressive than in white women with triple negative breast cancer $(2,3)$. To understand the transcriptional biology of triple negative breast cancer in an unbiased fashion and at the systems level, we mined published microarray data paired with patient survival data (1), performing comparative transcriptome analysis based on survival at time of data collection: dead or alive. We present here a finding from the preliminary results of this analysis.

\section{Methods}

We used dataset GSE142102 (1) for this differential gene expression analysis in conjunction with GEO2R. GSE142102 was generated using Affymetrix Human Gene 2.1 ST Array technology; in this analysis, we used data from $n=87$ tumors from TNBC patients that were dead at time of analysis and $n=123$ tumors from TNBC patients that were alive at time of analysis. The Benjamini and Hochberg method of $p$-value adjustment was used for ranking of differential expression but raw $p$-values were used to assess statistical significance of global differential expression. Log-transformation of data was auto-detected, and the NCBI generated category of platform annotation was used. A statistical test was performed to evaluate whether PARP15 expression was significantly different between the primary tumors of TNBC patients that were dead or alive using a two-tailed t-test. Data is graphically represented with standard error of the mean. For Kaplan-Meier survival analysis, we used the Kaplan-Meier plotter tool (4) for correlation of PARP15 mRNA expression levels with overall survival (OS) in $n=278$ patients with basal subtype cancer.

\section{Results}

We performed comparative transcriptome analysis using published microarray data (1) to understand in an unbiased fashion the most significant gene expression differences in the primary tumors of patients with triple negative breast cancer based on survival outcomes.

PARP15 is differentially expressed in triple negative breast cancer and positively correlates with survival.

When comparing primary tumors of 87 patients with triple negative breast cancer, alive at time of data collection and 123 TNBC patients, dead at time of data collection, we identified the poly (ADP-ribose) polymerase family, member 15, PARP15, as among the most significant transcriptional differences based on survival in human triple negative breast cancer. When ranking each of the transcripts whose expression was measured by microarray based on significance of difference in expression between the tumors of patients dead and alive, PARP15 ranked 223 out of 53617 total transcripts, equivalent to 99.6\% differential expression (Chart 1). PARP15 differential expression in the tumors of patients based on survival outcomes was statistically significant (Figure $1 ; p=0.00550407$ ). 
PARP15 is expressed at significantly higher levels in the breast tumors of TNBC patients when comparing based on survival.

We obtained exact mRNA expression levels for PARP15 from breast tumors of TNBC patients dead and alive to understand the magnitude and direction of PARP15 expression change based on survival outcomes. PARP15 was expressed at higher levels in tumors of TNBC patients alive as compared to that of TNBC patients dead (Figure 1). Increased expression of PARP15 in primary breast tumors was statistically significant (Figure 1: $p=0.0031$ ). PARP15 was expressed at $-0.19 \pm 0.69$ arbitrary units (AU) in the primary tumors of TNBC patients alive, while it was expressed at $0.20 \pm 1.18 \mathrm{AU}$ in the primary tumors of the TNBC patients dead.

\section{PARP15 expression correlates with overall survival in basal subtype human breast cancer.}

We referenced a bioinformatics tool containing Kaplan-Meier survival data of human breast cancer patients to understand more thoroughly whether a relationship existed between PARP15 primary tumor expression and patient survival in human breast cancer. We found that PARP15 expression was positively and significantly correlated with overall survival in patients with basal or basal-like human breast cancer, a breast cancer molecular subtype which shares significant overlap with triple negative breast cancer (Figure 2; log rank $p$-value: 0.002 for overall survival, hazard ratio: 0.47 (0.29-0.77) (Fig. 2)). PARP15 mRNA levels were a positive prognostic indicator in basal subtype breast cancer patients. Median OS was, on average, markedly shorter for basal subtype patients with low tumor expression of PARP15 as compared to basal subtype patients with high tumor expression of PARP15.

Thus, we found through unbiased comparative transcriptome analysis that PARP15 was among the genes whose expression was most significantly different in the primary tumors of triple negative breast cancer patients alive when compared to that of TNBC patients dead, that PARP15 was expressed at significantly higher levels in the primary tumors of TNBC patients alive as compared to TNBC patients dead, and that PARP15 expression was positively correlated with overall survival in the basal or basal-like molecular subtype.

\section{Discussion}

It is not understood why women of African descent (black women) are diagnosed with triple negative breast cancer more frequently than white women, and why this disease is more aggressive in black women. PARP15 may be of relevance as a biomarker in identifying patients who are more likely to survive, perhaps as part of a panel of genes used for prognostic stratification, and the molecule itself may be important to the biology of triple negative breast cancer etiology or more likely its progression. Blind systems-level analyses, like this, can help shed light on the fundamental molecular characteristics of a disease whose racial propensities demand further explanation. 


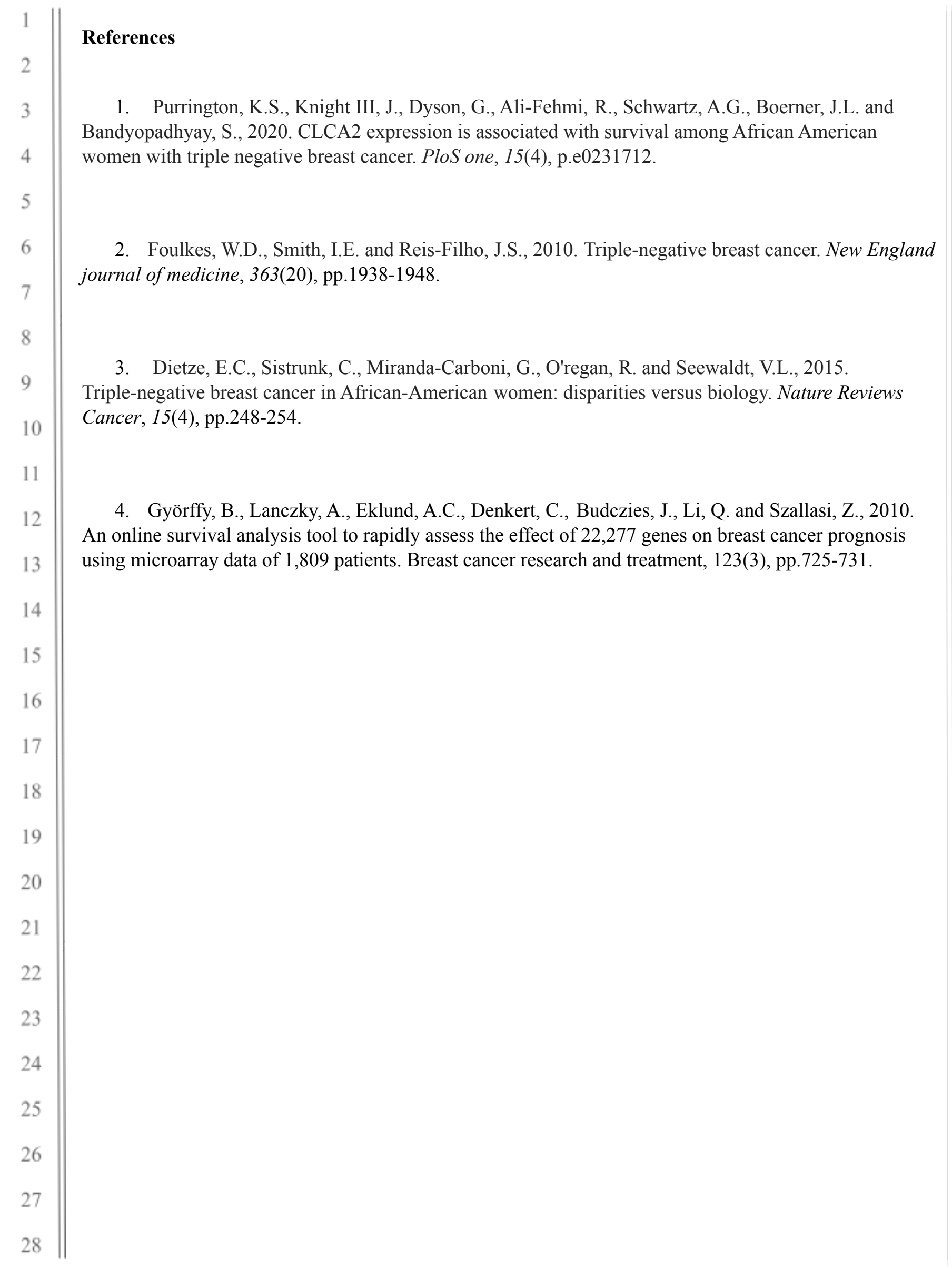




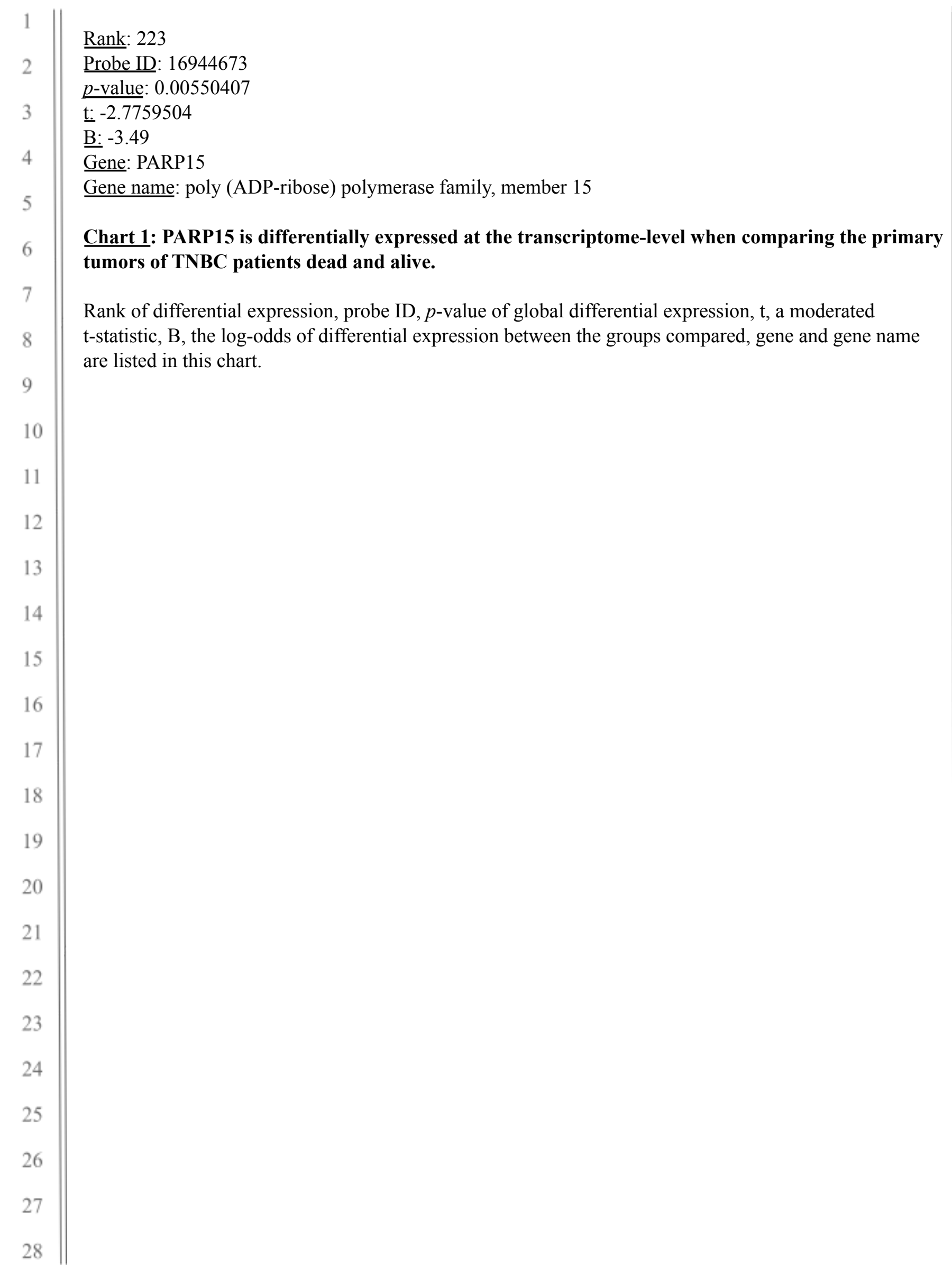




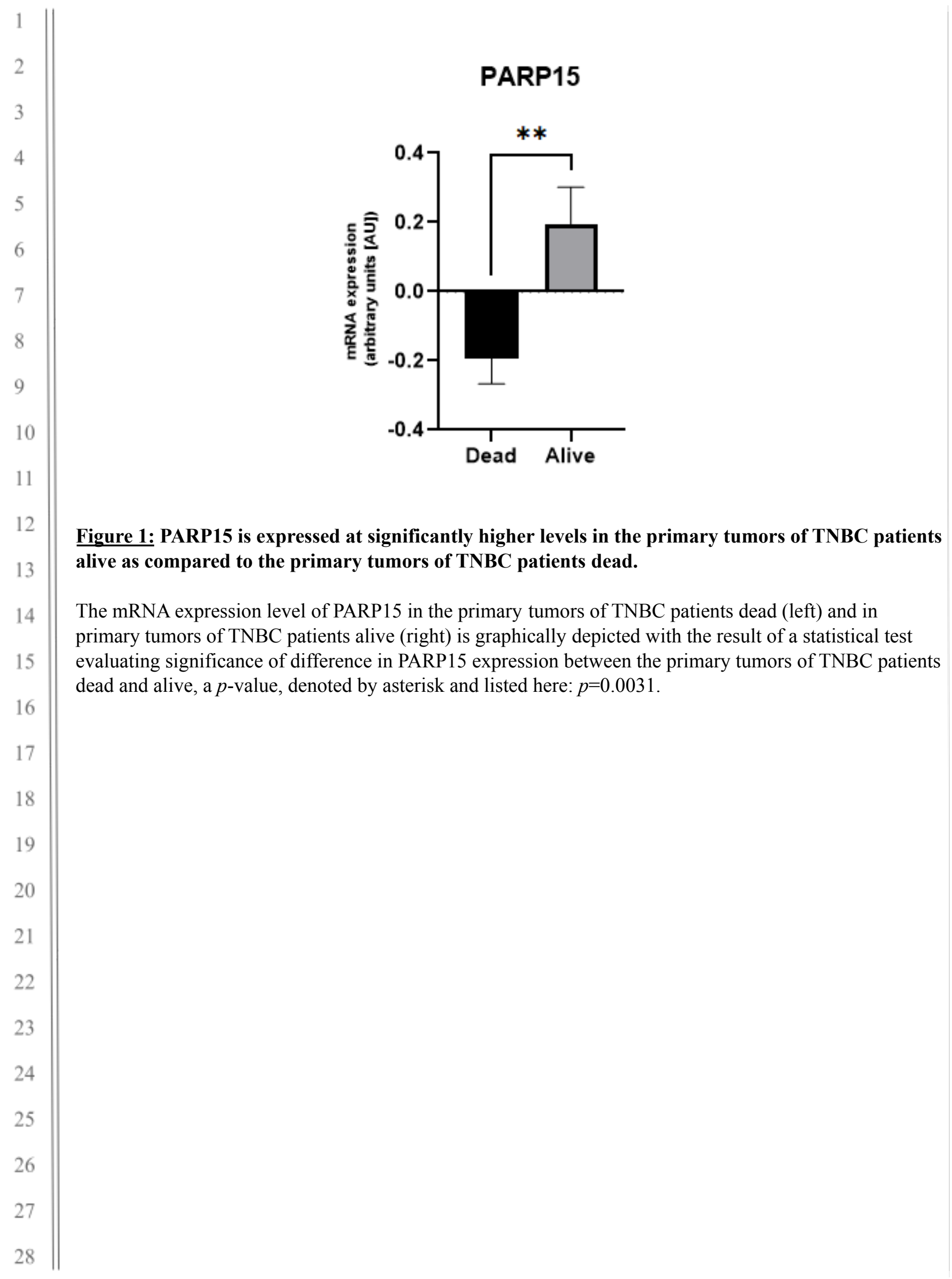




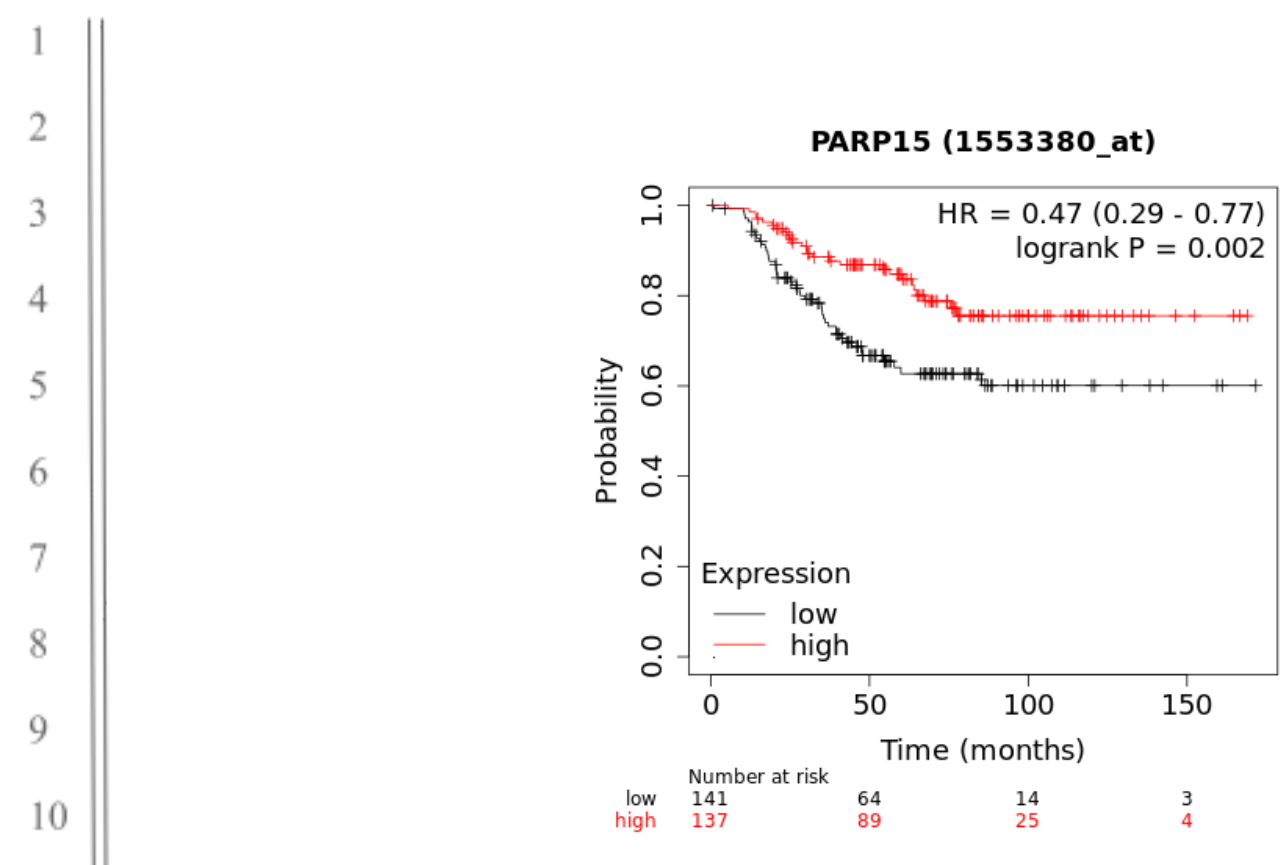

Figure 2: PARP15 expression correlates with overall survival in basal subtype human breast cancer.

Depicted in this Kaplan-Meier plot is the probability of overall survival (OS) for $n=278$ breast cancer patients of basal subtype, based on low or high expression of PARP15 in patient primary tumors. The log rank p-value denoting statistical significance of difference in recurrence-free survival when comparing the two groups, as well as hazard ratio for this comparison is listed above. Listed below is the number of patients at risk (number of patients alive) per interval, after stratification based on PARP15 expression; in the first interval, number at risk is number of patients alive; in each subsequent interval, number at risk is the number at risk less those who have expired or are censored. 